\title{
A UNIFIED APPROACH TO FOURIER-CLIFFORD-PROMETHEUS SEQUENCES, TRANSFORMS AND FILTER BANKS
}

Ekaterina L.-Rundblad, Valeriy Labunets, and Ilya Nikitin

Ural State Technical University

Ekaterinburg, Russia

lab@rtf.ustu.ru

\begin{abstract}
In this paper we develop a new unified approach to the so-called generalized Fourier-Clifford-Prometheus sequences, transforms (FCPTs) and $M$-channel Filter Banks. It is based on a new generalized FCPT-generating construction. This construction has a rich algebraic structure that supports a wide range of fast algorithms.
\end{abstract}

Keywords: Clifford algebra, filter banks, Golay-Shapiro sequences, Fourier-CliffordPrometheus transforms.

\section{Introduction}

The basis which has come to be known as the Prometheus Orthonormal Set (PONS) was introduced in [1] to prove the H.S. SHAPIRO global uncertainty principle conjecture. Each function in PONS is called a Golay-Shapiro sequence. They are defined on $[0,1]$, piecewise \pm 1 and can change sign only at points of the form $j / 2^{n}, j=0,1, \ldots, 2^{n}-1$, $n=1,2, \ldots$. These basis functions satisfy almost all standard properties of the Walsh functions. Discrete classical Fourier-Prometheus Transforms (FPT) in bases of different Golay-Shapiro sequences can be used in many signal processing applications: multiresolution by discrete orthogonal wavelet decomposition, digital audio, digital video broadcasting, communication systems (Orthogonal Frequency Division Multiplexing, Multi-Code Code-Division Multiple Access), radar, and cryptographic systems.

Golay-Shapiro (GS) 2-complementary $( \pm 1)$-valued sequences associated with the cyclic group $\mathbf{Z}_{2}$ were introduced by SHAPIRO and GolaY 
in 1949-1951 [2]-[7]. In 1961, Golay [3] gave an explicit construction for binary Golay complementary pairs of length $2^{m}$ and later noted [4] that the construction implies the existence of at least $2^{m} m ! / 2$ binary Golay sequences of this length. They are known to exist for all lengths $N=2^{\alpha} 10^{\beta} 26^{\gamma}$, where $\alpha, \beta, \gamma$ are integers and $\alpha, \beta, \gamma \geq 0$ [8], but do not exist for any length $N$ having a prime factor congruent to 3 modulo 4 [9]. Budisin [10] using the earlier work of Sivaswamy [11], gave a more general recursive construction for Golay complementary pairs and showed that the set of all binary Golay complementary pairs of length $2^{m}$ obtainable from it concides with those given explicitly by Golay [3]. For a survey of results on nonbinary Golay Complementary pairs, see [12][13]. Recently, DAVIS and JEDWAB [14], combining results appearing in the work of Golay and Shapiro cited above, gave an explicit description of a large class of Golay complementary sequences in terms of certain cosets of the first order Reed-Muller codes. The following general elements are used for building the classical Fourier-Prometheus transforms in bases of classical Golay-Shapiro sequences: 1) the Abelian group $\mathbf{Z}_{2}^{n}$, 2) the 2-point Fourier transform $\mathcal{F}_{2}$, and 3) the complex field C; i.e., these transforms are associated with the triple $\left(\mathbf{Z}_{2}^{n}, \mathcal{F}_{2}, \mathbf{C}\right)$.

The multiresolution analysis (MRA) operates upon a discrete signal $x(l)$ of length $2^{n}$, where $n$ is an integer. The sequence $x(l)$ is convolved with two filters $L$ and $H$. Each convolution results in a sequence half the length of the original sequence. The result from the convolution with the low-pass filter is again transformed. Each re-transformed sequence of the low-pass output is referred to as a dilation. For a sequence $x(l)$ of length $2^{n}$, a maximum of $n$ dilations can be performed. MRA applied to a real-valued sequence $x(l)$ is defined recursively by the equations:

$$
c^{(p)}(l)=L\left\{c^{(p-1)}(l)\right\}, \quad d^{(p)}=H\left\{c^{(p-1)(l)}\right\},
$$

where $p=n, n-1, \ldots, 1,0, c^{n}(l)=x(l)$, and

$$
\begin{aligned}
& c(l)=(L x)(l)=\sum_{l=0}^{2^{n}-1} k_{l p}(l-2 k) x(l), \\
& d(l)=(H x)(l)=\sum_{l=0}^{2^{n}-1} k_{h p}(l-2 k) x(l)
\end{aligned}
$$

are low-pass and high-pass filters, respectively.

The sequences $c^{(p)}(l)$ and $d^{(p)}(l)$ are called the "averages" and "differences" of the original signal. The inverse discrete wavelet transform reconstructs $c^{(n)}(l)=x(l)$ using the recursive algorithm

$$
c^{(p+1)}(l)=L^{*}\left\{c^{(p)}(l)\right\}+H^{*}\left\{d^{(p)}(l)\right\},
$$


where $L^{*}$ and $H^{*}$ are the inverse filters of $L$ and $H$, respectively. All filters $L, H$, and $L^{*}, H^{*}$, satisfy the following equation $L L^{*}=I, \quad H H^{*}=$ $I$, and

$$
L L^{*}+H H^{*}=2 I, \quad L H^{*}=H^{*} L=0,
$$

where $I$ and 0 denote the identity and zero operators. Note that a pair of filters having these properties required of the transformations $L$ and $H$ are known as quadrature mirror filters, having the perfect reconstruction property.

The conditions (1) can be rewritten in terms of the Z-transform as

$\left|k_{l p}(z)\right|^{2}+\left|k_{h p}(z)\right|^{2}=2, \quad \bar{k}_{l p}(z) k_{l p}(-z)+\bar{k}_{h p}(z) k_{h p}(-z)=0, \quad \forall z \in \mathbb{T}_{1}$

where $\mathbb{T}_{1}$ is the unit circle of the complex field $\mathbb{C}$. These conditions mean that impulse responses $k_{l p}(l)$ and $k_{h p}(l)$ form a Golay-Shapiro (GS) 2complementary pair.

In this paper we develop a new unified approach to the so-called generalized Fourier-Clifford-Prometheus (FCP) sequences, FCP transforms (FCPTs), and M-channel Filter Banks. We describe the precise theoretical and computational relationship between $M$-band wavelets, $M$-channel filterbanks and generalized Golay-Shapiro sequences. The approach is based on a new generalized FCPT-generating construction. This construction has a rich algebraic structure that supports a wide range of fast algorithms. This construction is associated not with the

triple $\left(\mathbf{Z}_{2}^{n}, \mathcal{F}_{2}, \mathbf{C}\right)$, but rather with other groups instead of $\mathbf{Z}_{2}^{n}$, other unitary transforms instead of $\mathcal{F}_{2}$, and other algebras (Clifford algebras) instead of the complex field $\mathbf{C}$.

\section{New construction of classical and multiparametric Prometheus transforms}

We begin by describing the original Golay 2-complementary $( \pm 1)$ valued sequences.

Definition 1 Let $\mathbf{p}(t):=\left(p_{0}, p_{1}, \ldots, p_{N-1}\right), \mathbf{q}(t):=\left(q_{0}, q_{1}, \ldots, q_{N-1}\right)$, where $p_{i}, q_{i} \in\{ \pm 1\}$. The sequences $\mathbf{p}(t), \mathbf{q}(t)$ are called a 2-complementary $( \pm 1)$-valued or Golay complementary pair over $\{ \pm 1\}$ if

$$
\operatorname{COR}[\mathbf{p}, \mathbf{p}](\tau)+\operatorname{COR}[\mathbf{q}, \mathbf{q}](\tau)=N \delta(\tau),
$$

or

$$
|\mathbf{p}(z)|^{2}+|\mathbf{q}(z)|^{2}=N, \quad \forall z \in \mathbb{T}_{1},
$$

where $\operatorname{COR}[\mathbf{f}, \mathbf{f}](\tau)$ is the periodic correlation function of $\mathbf{f}(t) ; \mathbf{p}(z)$ and $\mathbf{q}(z)$ are $z$-transforms of $\mathbf{p}(t)$ and $\mathbf{q}(t)$, respectively. Any sequence which is a member of a Golay complementary pair is called a Golay sequence. 
The Fourier-Prometheus matrix of depth $n$ has size $2^{n} \times 2^{n}: \mathcal{F P}_{2^{n}}=$ $\left[\operatorname{Pr}_{\alpha}(t)\right]_{\alpha, t=0}^{2^{n}-1}$. For $\alpha$ and $t$ we shall use binary representations $\alpha=\alpha_{[n]}:=$ $\left(\alpha_{1}, \alpha_{2}, \ldots, \alpha_{n}\right), t=t_{[n]}:=\left(t_{1}, t_{2}, \ldots, t_{n}\right)$, where $\alpha_{i}, t_{i} \in\{0,1\}, i=$ $1,2, \ldots, n$. Obviously, $\alpha_{[1]}=\left(\alpha_{1}\right), \alpha_{[2]}=\left(\alpha_{1}, \alpha_{2}\right), \alpha_{[3]}=\left(\alpha_{1}, \alpha_{2}, \alpha_{3}\right), \ldots$ $t_{[1]}=\left(t_{1}\right), t_{[2]}=\left(t_{1}, t_{2}\right), t_{[n]}=\left(t_{1}, t_{2}, \ldots, t_{n}\right), \ldots$ For this reason,

$$
2^{n} \mathcal{F} \mathcal{P}_{\left(\alpha_{[n-1]}, \alpha_{n}\right)}=\left[\begin{array}{l}
\operatorname{Pr}_{(0,0, \ldots, 0,0)}\left(t_{1}, \ldots, t_{n}\right) \\
\operatorname{Pr}_{(0,0, \ldots, 0,1)}\left(t_{1}, \ldots, t_{n}\right) \\
\hline \operatorname{Pr}_{(0,0, \ldots, 1,0)}\left(t_{1}, \ldots, t_{n}\right) \\
\operatorname{Pr}_{(0,0, \ldots, 1,1)}\left(t_{1}, \ldots, t_{n}\right) \\
\hline \ldots \\
\ldots \\
\hline \operatorname{Pr}_{(1,1, \ldots, 1,0}\left(t_{1}, \ldots, t_{n}\right) \\
\operatorname{Pr}_{(1,1, \ldots, 1,1}\left(t_{1}, \ldots, t_{n}\right)
\end{array}\right]=\boxplus_{\alpha_{[n-1]}=0}^{2^{n-1}-1}\left[\begin{array}{l}
\operatorname{Pr}_{\left(\alpha_{[n-1]}, 0\right)}(t) \\
\operatorname{Pr}_{\left(\alpha_{[n-1]}, 1\right)}(t)
\end{array}\right]
$$

where $\operatorname{Pr}_{\left(\alpha_{[n-1]}, 0\right)}(t)$ and $\operatorname{Pr}_{\left(\alpha_{[n-1]}, 1\right)}(t)$ are a pair of GS 2-complementary sequences and $\boxplus$ represents the vertical concatenation of matrices.

The classical matrix $\mathcal{F P}_{2^{n}}$ is formed by starting with the $(2 \times 2)$ matrix ${ }^{2} \mathcal{F P}=\left[\begin{array}{l}\operatorname{Pr}_{0}(t) \\ \operatorname{Pr}_{1}(t)\end{array}\right]=\left[\begin{array}{rr}1 & 1 \\ 1 & -1\end{array}\right]$ and by repeated application of the PONS-iteration construction to pairs of rows in the matrix. In the $(n+1)$ st iteration this construction takes each pair $\left[\begin{array}{l}\mathbf{p} \\ \mathbf{q}\end{array}\right]=$

$$
\begin{aligned}
& {\left[\begin{array}{l}
\operatorname{Pr}_{\left(\alpha_{[n-1]}, 0\right)}(t) \\
\operatorname{Pr}_{\left(\alpha_{[n-1]}, 1\right)}(t)
\end{array}\right] \text { of }} \\
& { }^{2^{n}} \mathcal{F P}\left(\alpha_{[n-1]}, \alpha_{n}\right)=\boxplus_{\alpha_{[n-1]}=0}^{2^{n-1}-1}\left[\begin{array}{l}
\operatorname{Pr}_{\left(\alpha_{[n-1]}, 0\right)}(t) \\
\operatorname{Pr}_{\left(\alpha_{[n-1]}, 1\right)}(t)
\end{array}\right]
\end{aligned}
$$

and constructs four rows of twice the length

$$
\begin{gathered}
\operatorname{PONS}(\mathbf{p}, \mathbf{q})=\left[\begin{array}{rr}
\mathbf{p} & \mathbf{q} \\
\mathbf{p} & -\mathbf{q} \\
\hline \mathbf{q} & \mathbf{p} \\
-\mathbf{q} & -\mathbf{p}
\end{array}\right]=\left[\begin{array}{rr}
\mathbf{p} & \mathbf{q} \\
\mathbf{p} & -\mathbf{q}
\end{array}\right] \boxplus\left[\begin{array}{rr}
\mathbf{q} & \mathbf{p} \\
-\mathbf{q} & -\mathbf{p}
\end{array}\right] \\
=\left(\left[\begin{array}{rr}
1 & 1 \\
1 & -1
\end{array}\right]\left[\begin{array}{l|r}
\mathbf{p} & \\
\hline & \mathbf{q}
\end{array}\right]\right) \boxplus\left(\left[\begin{array}{rr}
1 & 1 \\
1 & -1
\end{array}\right]\left[\begin{array}{l|l} 
& \mathbf{p} \\
\hline \mathbf{q} &
\end{array}\right]\right) \\
=\left(\left[\begin{array}{rr}
1 & 1 \\
1 & -1
\end{array}\right]\left[\begin{array}{l|l|l|}
\mathbf{p} & \\
\hline & \mathbf{q}
\end{array}\right]\left[\begin{array}{ll}
1 & 1
\end{array}\right]\right) \boxplus\left(\left[\begin{array}{rr}
1 & 1 \\
1 & -1
\end{array}\right]\left[\begin{array}{l|l}
\mathbf{p} & \\
\hline & \mathbf{q}
\end{array}\right]\left[\begin{array}{ll}
1 & 1
\end{array}\right]\right) \\
=\left(\mathcal{F}_{2}\left[\begin{array}{l|l|l}
\mathbf{p} & \\
\hline & \mathbf{q}
\end{array}\right] T_{2}^{0}\right) \boxplus\left(\mathcal{F}_{2}\left[\begin{array}{l|l}
\mathbf{p} & \\
\hline & \mathbf{q}
\end{array}\right] T_{2}^{1}\right),
\end{gathered}
$$


where $\left\{T^{\alpha_{1}}\right\}_{\alpha_{1}=0}^{1}$ are dyadic shifts. Using this construction for all $2^{k-2}$ complementary pairs $\left(\alpha_{[k-2]}=0,1, \ldots, 2^{k-2}-1\right)$, we obtain

$$
\begin{aligned}
& 2^{n+1} \mathcal{F} \mathcal{P}_{\left(\alpha_{[n]}, \alpha_{n+1}\right)}=\underset{\alpha_{[n]}=0}{\boxplus^{2^{n}}-1}\left(\mathcal{F}_{2}\left[\begin{array}{l|l}
\mathbf{p} & \\
\hline & \mathbf{q}
\end{array}\right] T_{2}^{\alpha_{n}}\right) \\
= & \underset{\alpha_{[n]}=0}{\boxplus^{n}-1}\left(\mathcal{F}_{2}\left[\begin{array}{l|l}
\operatorname{Pr}_{\left(\alpha_{[n-1]}, 0\right)}(t) & \\
\hline & \operatorname{Pr}_{\left(\alpha_{[n-1]}, 1\right)}(t)
\end{array}\right] T_{2}^{\alpha_{n}}\right) .
\end{aligned}
$$

Repetition of this construction yields the Fourier-Prometheus matrix $2^{n+1} \mathcal{F P}$ of size $2^{n+1} \times 2^{n+1}$.

Our new PONS construction uses in (2) three parametric unitary matrices

$$
\mathcal{U}_{2}(\beta, \varphi, \gamma)=\left[\begin{array}{rr}
e^{i(\beta+\gamma)} \cos \varphi & e^{i(\beta-\gamma)} \sin \varphi \\
e^{-i(\beta-\gamma)} \sin \varphi & -e^{-i(\beta+\gamma)} \cos \varphi
\end{array}\right]
$$

instead of $\mathcal{F}_{2}$ :

$$
\begin{aligned}
& 2^{n+1} \mathcal{F P}\left(\alpha_{[n]}, \alpha_{n+1}\right) \\
& \left.*\left[\begin{array}{l|l}
\left.\operatorname{Pr}_{n+1}, \vec{\varphi}_{n+1}, \vec{\gamma}_{n+1}\right)=\boxplus_{\left.\alpha_{[n]}, 0\right)}^{2^{n}-1}\left(t \mid \vec{\beta}_{n}, \vec{\varphi}_{n}, \vec{\gamma}_{n}\right) & \\
\hline & \operatorname{Pr}_{\left(\alpha_{[n-1]}, 1\right)}\left(t \mid \vec{\beta}_{n}, \vec{\varphi}_{n}, \vec{\gamma}_{n}\right)
\end{array}\right] T_{2}^{\alpha_{k}}\right)
\end{aligned}
$$

where

$$
\vec{\beta}_{n+1}=\left(\beta_{1}, \ldots, \beta_{n+1}\right), \vec{\varphi}_{n+1}=\left(\varphi_{1}, \ldots, \varphi_{n+1}\right), \vec{\gamma}_{n+1}=\left(\gamma_{1}, \ldots, \gamma_{n+1}\right)
$$

are three $(n+1) \mathrm{D}$ vectors of parameters. Extra parameters $\beta_{k}, \varphi_{k}, \gamma_{k}$ $(k=1,2, \ldots, n+1)$ are changed from stage to stage in this construction. The resulting matrix still has orthogonal rows and every pair is 2-complementary in the Golay-Shapiro sense.

\section{PONS associated with Abelian groups}

\section{$3.1 \quad$ Abelian groups $\mathrm{Z}_{N}^{n}$}

A natural generalization of a 2-complementary Golay pair is an $N$ complementary Golay $N$-member orthogonal set of Clifford-valued sequences $\mathbf{p}_{0}(t), \ldots, \mathbf{p}_{N-1}(t)$, where $t=0,1, \ldots, N^{n}-1$.

Definition 2 Let $\mathbf{p}_{0}(t), \mathbf{p}_{1}(t), \ldots, \mathbf{p}_{N-1}(t)$ be an $N$-member orthogonal set of Clifford-valued sequences, where $\mathbf{p}_{i}(t) \in\left\{\varepsilon_{N}^{k}\right\}_{k=0}^{N-1}, \varepsilon_{N}:=$ 
$e^{2 \pi \mathbf{u} / N} \in$ Cla, Cla is a Clifford algebra, and $\mathbf{u}$ is an appropriate bivector with the property $\mathbf{u}^{2}=-1$. The sequences $\left\{\mathbf{p}_{i}(t)\right\}_{i=0}^{N-1}$ are called $N$-complementary $\left\{\varepsilon_{N}^{k}\right\}_{k=0}^{N-1}$-valued sequences of length $N^{n}$ if

$$
\operatorname{COR}\left[\mathbf{p}_{0}, \mathbf{p}_{0}\right](\tau)+\ldots+\operatorname{COR}\left[\mathbf{p}_{N-1}, \mathbf{p}_{N-1}\right](\tau)=N^{n} \delta(\tau),
$$

or $\left|\mathbf{p}_{0}(z)\right|^{2}+\left|\mathbf{p}_{1}(z)\right|^{2}+\ldots+\left|\mathbf{p}_{N-1}(z)\right|^{2}=N^{n}, \quad \forall z \in \mathbb{T}_{1}$, where $\mathbf{p}_{i}(z)$ are $z$-transforms of $\mathbf{p}_{i}(t), i=0,1, \ldots, N-1$, respectively.

Let, for example, $N=3$. Then for the group $\mathbf{Z}_{3}$ we define the FourierClifford-Prometheus transform as the Fourier-Clifford transform

$$
{ }^{3} \mathcal{F C P}:={ }^{1} \mathcal{F} \mathcal{C}=\left[\begin{array}{l}
\operatorname{Pr}_{0}(t) \\
\operatorname{Pr}_{1}(t) \\
\operatorname{Pr}_{2}(t)
\end{array}\right]=\left[\begin{array}{lll}
1 & 1 & 1 \\
1 & \varepsilon_{3} & \varepsilon_{3}^{2} \\
1 & \varepsilon_{3}^{2} & \varepsilon_{3}
\end{array}\right] .
$$

For the group $\mathbf{Z}_{3}^{2}$ we define the Fourier-Clifford-Prometheus transform using the classical PONS-construction (2) by

$$
\begin{aligned}
& 3^{2} \mathcal{F} \mathcal{P}_{\alpha_{1}, \alpha_{2}}=\boxplus_{\alpha_{1}=0}^{2}\left(\mathcal{F e}_{3}\left[\begin{array}{l|l|l}
\operatorname{Pr}_{0}(t) & & \\
\hline & \operatorname{Pr}_{1}(t) & \\
\hline & & \operatorname{Pr}_{2}(t)
\end{array}\right] T_{3}^{\alpha_{1}}\right)
\end{aligned}
$$

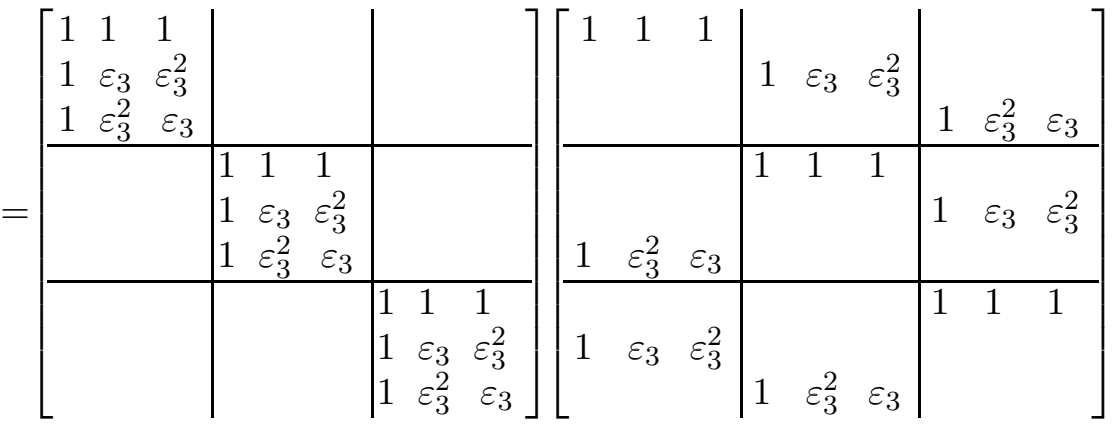

$$
\begin{aligned}
& =\left[\begin{array}{lll|lll|lll}
1 & 1 & 1 & 1 & \varepsilon_{3} & \varepsilon_{3}^{2} & 1 & \varepsilon_{3}^{2} & \varepsilon_{3} \\
1 & 1 & 1 & \varepsilon_{3} & \varepsilon_{3}^{2} & 1 & \varepsilon_{3}^{2} & \varepsilon_{3} & 1 \\
1 & 1 & 1 & \varepsilon_{3}^{2} & 1 & \varepsilon_{3} & \varepsilon_{3} & 1 & \varepsilon_{3}^{2} \\
\hline 1 & \varepsilon_{3}^{2} & \varepsilon_{3} & 1 & 1 & 1 & 1 & \varepsilon_{3} & \varepsilon_{3}^{2} \\
\varepsilon_{3}^{2} & \varepsilon_{3} & 1 & 1 & 1 & 1 & \varepsilon_{3} & \varepsilon_{3}^{2} & 1 \\
\varepsilon_{3} & 1 & \varepsilon_{3}^{2} & 1 & 1 & 1 & \varepsilon_{3}^{2} & 1 & \varepsilon_{3} \\
\hline 1 & \varepsilon_{3} & \varepsilon_{3}^{2} & 1 & \varepsilon_{3}^{2} & \varepsilon_{3} & 1 & 1 & 1 \\
\varepsilon_{3} & \varepsilon_{3}^{2} & 1 & \varepsilon_{3}^{2} & \varepsilon_{3} & 1 & 1 & 1 & 1 \\
\varepsilon_{3}^{2} & 1 & \varepsilon_{3} & \varepsilon_{3} & 1 & \varepsilon_{3}^{2} & 1 & 1 & 1
\end{array}\right]=\left[\begin{array}{l}
\operatorname{Pr}_{(0,0)}(t) \\
\operatorname{Pr}_{(0,1)}(t) \\
\operatorname{Pr}_{(0,2)}(t) \\
\hline \operatorname{Pr}_{(1,0)}(t) \\
\operatorname{Pr}_{(1,1)}(t) \\
\operatorname{Pr}_{(1,2)}(t) \\
\hline \operatorname{Pr}_{(2,0)}(t) \\
\operatorname{Pr}_{(2,1)}(t) \\
\operatorname{Pr}_{(2,2)}(t)
\end{array}\right]
\end{aligned}
$$

where $\left\{T^{\alpha_{1}}\right\}_{\alpha_{1}=0}^{2}$ are 3 -cyclic shift operators. After $n+1$ iterations we obtain the following Fourier-Clifford-Prometheus transform on the group 
$\mathbf{Z}_{3}^{n+1}$ :

$$
3^{n+1} \mathcal{F} \mathcal{P P}\left(\alpha_{[n]}, \alpha_{n+1}\right)=\underset{\alpha_{[n]}=0}{\boxplus 3^{n}-1}\left(\mathcal{F e}_{3}\right.
$$

$\left.*\left[\begin{array}{l|l|l}\operatorname{Pr}_{\left(\alpha_{[n-1]}, 0\right)}(t) & & \\ \hline & \operatorname{Pr}_{\left(\alpha_{[n-1]}, 1\right)}(t) & \\ \hline & & \operatorname{Pr}_{\left(\alpha_{[n-1]}, 2\right)}(t)\end{array}\right] T_{3}^{\alpha_{n}}\right)$.

The same expression is true for the Fourier-Clifford-Prometheus transform on the group $\mathbf{Z}_{N}^{n}$ :

$$
N^{n+1} \mathcal{F} \mathcal{P} \mathcal{P}_{\left(\alpha_{[n]}, \alpha_{n+1}\right)}=\underset{\alpha_{[n]}=0}{\boxplus^{[n]}-1}\left(\mathcal{F} \mathcal{C}_{N}\right.
$$

$\left.*\left[\begin{array}{l|l|l|l}\operatorname{Pr}_{\left(\alpha_{[n-1]}, 0\right)}(t) & & & \\ \hline & \operatorname{Pr}_{\left(\alpha_{[n-1]}, 1\right)}(t) & & \\ \hline & & \ddots & \\ \hline & & & \operatorname{Pr}_{\left(\alpha_{[n-1]}, N-1\right)}(t)\end{array}\right] T_{N}^{\alpha_{n}}\right)$,

where $\mathcal{F C}_{N}$ is the Fourier-Clifford transform on the group $\mathbf{Z}_{N}$,

$$
\left\{T^{\alpha_{1}}\right\}_{\alpha_{1}=0}^{N-1}
$$

are $N$-cyclic shift operators.

\subsection{Abelian groups $\mathbf{Z}_{N_{1}} \oplus \mathbf{Z}_{N_{2}} \oplus \ldots \oplus \mathbf{Z}_{N_{n}}$}

Let $\mathbf{Z}_{N_{1}} \oplus \mathbf{Z}_{N_{2}} \oplus \ldots \oplus \mathbf{Z}_{N_{n}}$ be an Abelian group, where $N_{1}, N_{2}, \ldots, N_{n}$ are positive integers. The classical Fourier-Prometheus transforms are generated by the Fourier-Walsh transform $\mathcal{F}_{2}$ and by dyadic shifts. Fourier-Clifford-Prometheus transforms associated with $\mathbf{Z}_{N}^{n}$ are generated by the Fourier-Clifford transform $\mathcal{F C}_{N}$ of the group $\mathbf{Z}_{N}$ and by $N$-ary shifts. We shall generate new Fourier-Clifford-Prometheus transforms associated with Abelian groups $\mathbf{Z}_{N_{1}} \oplus \mathbf{Z}_{N_{2}} \oplus \ldots \oplus \mathbf{Z}_{N_{n}} \oplus \mathbf{Z}_{N_{n+1}}$ by using the set of Fourier-Clifford transforms $\mathcal{F C}_{N_{1}}, \mathcal{F C}_{N_{2}}, \ldots, \mathcal{F C}_{N_{n}}, \mathcal{F C}_{N_{n+1}}$. For example, the group $\mathbf{Z}_{2} \oplus \mathbf{Z}_{3} \oplus \mathbf{Z}_{4}$ requires three Fourier-Clifford transforms

$$
\mathcal{F}_{2}=\left[\begin{array}{rr}
1 & 1 \\
1 & -1
\end{array}\right], \quad \mathcal{F}_{3}=\left[\begin{array}{lll}
1 & 1 & 1 \\
1 & \varepsilon_{3} & \varepsilon_{3}^{2} \\
1 & \varepsilon_{3}^{2} & \varepsilon_{3}
\end{array}\right], \quad \mathcal{F}_{4}=\left[\begin{array}{llll}
1 & 1 & 1 & 1 \\
1 & \varepsilon_{4}^{1} & \varepsilon_{4}^{2} & \varepsilon_{4}^{3} \\
1 & \varepsilon_{4}^{2} & 1 & \varepsilon_{4}^{2} \\
1 & \varepsilon_{4}^{3} & \varepsilon_{4}^{2} & \varepsilon_{4}^{1}
\end{array}\right] .
$$


Let us consider the group $\mathbf{Z}_{2} \oplus \mathbf{Z}_{3}$. $\mathcal{F} \mathcal{E} \mathcal{P}_{2}=\mathcal{F e}_{2}=\left[\begin{array}{c}\operatorname{Pr}_{0}(t) \\ \operatorname{Pr}_{1}(t)\end{array}\right]=$ $\left[\begin{array}{rr}1 & 1 \\ 1 & -1\end{array}\right]$. We define the Fourier-Clifford-Prometheus transform associated with the Abelian group $\mathbf{Z}_{2} \oplus \mathbf{Z}_{3}$ by using the classical PONS construction

$$
\begin{aligned}
& { }^{2 \cdot 3} \mathcal{F} \mathcal{C P}_{\left(\alpha_{1}, \alpha_{2}\right)} \\
& =\boxplus_{\alpha_{1}=0}^{1}\left(\mathcal{F e}_{3}\left[\begin{array}{l|l|l}
\operatorname{Pr}_{\left\langle\left(\alpha_{1}, 0\right)\right\rangle_{2}}(t) & & \\
\hline & \operatorname{Pr}_{\left\langle\left(\alpha_{1}, 1\right)\right\rangle_{2}}(t) & \\
\hline & & \operatorname{Pr}_{\left\langle\left(\alpha_{1}, 2\right)\right\rangle_{2}}(t)
\end{array}\right] T_{3}^{\alpha_{1}}\right),
\end{aligned}
$$

where $\left\langle\left(\alpha_{1}, \beta_{2}\right)\right\rangle_{2}:=\left(\alpha_{1}, \beta_{2}\right) \bmod 2$. Therefore,

$$
\begin{aligned}
& \mathcal{F e P}_{2 \cdot 3}=\left[\begin{array}{ccc|ccc|cc|c}
1 & 1 & 1 & & & \\
1 & \varepsilon_{3} & \varepsilon_{3}^{2} & & & \\
1 & \varepsilon_{3}^{2} & \varepsilon_{3} & & & \\
\hline & & & 1 & 1 & 1 \\
& & & 1 & \varepsilon_{3} & \varepsilon_{3}^{2} \\
& & & 1 & \varepsilon_{3}^{2} & \varepsilon_{3}
\end{array}\right]\left[\begin{array}{cc|cc|cc}
1 & 1 & & & \\
& & 1 & -1 & & \\
& & & & 1 & 1 \\
\hline & & 1 & 1 & & \\
1 & -1 & & & &
\end{array}\right] \\
& =\left[\begin{array}{rr|rr|rr}
1 & 1 & 1 & -1 & 1 & 1 \\
1 & 1 & \varepsilon_{3} & \varepsilon_{3} & \varepsilon_{3}^{2} & \varepsilon_{3}^{2} \\
1 & 1 & \varepsilon_{3} & -\varepsilon_{3} & \varepsilon_{3}^{2} & \varepsilon_{3}^{2} \\
\hline 1 & -1 & 1 & 1 & 1 & -1 \\
\varepsilon_{3}^{2} & -\varepsilon_{3}^{2} & 1 & 1 & \varepsilon_{3} & -\varepsilon_{3} \\
\varepsilon_{3} & -\varepsilon_{3} & 1 & 1 & \varepsilon_{3}^{2} & -\varepsilon_{3}^{2}
\end{array}\right]=\left[\begin{array}{l}
\operatorname{Pr}_{(0,0)}(t) \\
\operatorname{Pr}_{(0,1)}(t) \\
\operatorname{Pr}_{(0,2)}(t) \\
\hline \operatorname{Pr}_{(1,0)}(t) \\
\operatorname{Pr}_{(1,1)}(t) \\
\operatorname{Pr}_{(1,2)}(t)
\end{array}\right] .
\end{aligned}
$$

We design Fourier-Clifford-Prometheus transforms associated with the Abelian groups $\mathbf{Z}_{N_{1}} \oplus \mathbf{Z}_{N_{2}} \oplus \ldots \oplus \mathbf{Z}_{N_{n+1}}$ by the same classical PONS construction

$$
\begin{gathered}
N^{[n+1]} \mathcal{F e P}_{\left(\alpha_{[n]} \alpha_{n+1}\right)} \\
=\boxplus_{\alpha_{[n]}=0}^{N^{[n]}-1}\left(\mathcal{F C}_{N_{n+1}}\left[\begin{array}{l|l|l}
\operatorname{Pr}_{\left(\alpha_{[n-1]},\langle 0\rangle_{N_{n}}\right)} & & \\
\hline & \ddots & \\
\hline & & \operatorname{Pr}_{\left(\alpha^{(n-1)},\left\langle N_{n+1}-1\right\rangle_{N_{n}}\right)}
\end{array}\right] T_{N_{n+1}}^{\alpha_{n}}\right)
\end{gathered}
$$

where $\mathcal{F}_{N_{n+1}}$ is the Fourier-Clifford transform on the group $\mathbf{Z}_{N_{n+1}},\left\langle\alpha_{n}+\right.$ $\left.\beta_{n+1}\right\rangle_{n+1}:=\left(\alpha_{n}+\beta_{n+1}\right) \bmod N_{n+1}, \alpha_{[n]}:=\left(\alpha_{1}, \alpha_{2}, \ldots, \alpha_{n}\right), N^{[n]}:=$ $N_{1} N_{2} \cdots N_{n},\left(\alpha_{[n]}, \beta_{n+1}\right):=\left(\alpha_{1}, \ldots, \alpha_{n}, \beta_{n+1}\right)$, and, hence,

$$
\left\langle\left(\alpha_{[n]}, \beta_{n+1}\right)\right\rangle_{n}:=\left(\alpha_{1}, \ldots, \alpha_{n}, \beta_{n+1}\right) \bmod N_{n} .
$$




\section{Fast Fourier-Prometheus Transforms \\ 4.1 Radix-2 Fast Transforms}

Let us return to the Fourier-Clifford-Prometheus transform

$$
\begin{aligned}
& \mathcal{F P}_{2^{2}}=\left[\begin{array}{l}
\operatorname{Pr}_{(0,0)}(t) \\
\operatorname{Pr}_{(0,1)}(t) \\
\hline \operatorname{Pr}_{(1,0)}(t) \\
\operatorname{Pr}_{(1,1)}(t)
\end{array}\right]=\left[\begin{array}{rr|rr}
1 & 1 & 1 & -1 \\
1 & 1 & -1 & 1 \\
\hline 1 & -1 & 1 & 1 \\
-1 & 1 & 1 & 1
\end{array}\right]
\end{aligned}
$$

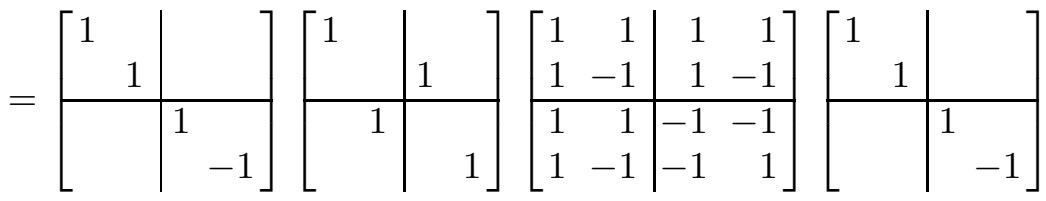

$$
\begin{aligned}
& =\Delta_{0} \Pi_{4}\left(\mathcal{F}_{2} \otimes \mathcal{F}_{2}\right) \Delta_{0},
\end{aligned}
$$

where $\Delta_{0}:=\operatorname{diag}\left(\operatorname{Pr}_{0}(t)\right)=\operatorname{diag}\left(\operatorname{Pr}_{\alpha}(0)\right)$ is a diagonal matrix and $\Pi_{4}$ is a special permutation matrix. From this expression we see that Prometheus functions up to constant factor are modulated Walsh functions:

$$
\operatorname{Pr}_{\left(\alpha_{1}, \alpha_{2}\right)}\left(t_{1}, t_{2}\right)=(-1)^{\alpha_{1} \alpha_{2}}\left[\operatorname{Wal}_{\left(\alpha_{1}, \alpha_{2}\right)}\left(t_{1}, t_{2}\right)(-1)^{t_{1} t_{2}}\right],
$$

where $(-1)^{\alpha_{1} \alpha_{2}}$ and $(-1)^{t_{1} t_{2}}$ are the so-called Shapiro multipliers, and $\mathrm{Wal}_{\left(\alpha_{1}, \alpha_{1}\right)}\left(t_{1}, t_{2}\right)=(-1)^{\alpha_{1} t_{1} \oplus_{2} \alpha_{2} t_{2}}$. The same result is true in the general case for the Fourier-Clifford-Prometheus $\left(2^{n} \times 2^{n}\right)$-transform $\mathcal{F P}_{2^{n}}=$ $\Delta_{0} \Pi_{2^{n}}\left(\mathcal{F}_{2} \otimes \mathcal{F}_{2} \otimes \cdots \otimes \mathcal{F}_{2}\right) \Delta_{0}$, where $\Pi_{2^{n}}$ is a special permutation matrix and $\Delta_{0}=\operatorname{diag}\left(\operatorname{Pr}_{0}(t)\right)=\operatorname{diag}\left(\operatorname{Pr}_{\alpha}(0)\right)$ is the diagonal matrix whose diagonal elements form the Shapiro $( \pm 1)$-multipliers. If $\alpha=\left(\alpha_{1}, \alpha_{2}, \ldots, \alpha_{n}\right)$ is the binary representation of the number in the $\alpha$ th row of $\Delta_{0}$, where $\alpha_{i} \in \mathbf{Z}_{2}$, then for diagonal elements $\Delta_{\alpha, \alpha}$ we have the expression $\Delta_{\alpha, \alpha}=$ $(-1)^{\sum_{i=1}^{n-1} \alpha_{i} \alpha_{i+1}}$. The quantity $b(\alpha)=\sum_{i=1}^{n-1} \alpha_{i} \alpha_{i+1}$ is the number of occurrences of the block $B=(11)$ in the binary representation of $\alpha$, $\left(\alpha_{1}, \alpha_{2}, \ldots, \alpha_{n}\right)$. For this reason the Fourier-Clifford-Prometheus transform has the Cooley-Tukey fast algorithm

$$
\mathcal{F P}_{2^{n}}=\Delta_{0} \Pi_{2^{n}}\left[C T_{2^{n}}^{1} C T_{2^{n}}^{2} \cdots C T_{2^{n}}^{n}\right] \Delta_{0},
$$

where $C T_{2^{n}}^{i}:=I_{2} \otimes \ldots \otimes \mathcal{F}_{2} \otimes \ldots \otimes I_{2}$ for $i=1,2, \ldots, n$ are the so-called Cooley-Tukey sparse matrices.

Now we can prove that an analogous result is true for Davis-Jedwab Clifford-valued sequences. Let $\mathbf{M C}_{2^{h}}=\left\{\varepsilon_{2^{h}}^{k}\right\}_{k=0}^{2^{h}-1}$ be the multiplicative cyclic group of $2^{h}$ th roots of unity and $\varepsilon_{2^{h}}$ be a $2^{h}$ th primitive root in a 
Clifford algebra Cla. Let $\left(c_{1}, c_{2}, \ldots, c_{n}\right) \in \mathbf{Z}_{2^{h}}^{n}=\mathbf{Z}_{2^{h}} \oplus \mathbf{Z}_{2^{h}} \oplus \ldots \oplus \mathbf{Z}_{2^{h}}$, be an $n \mathrm{D}$ vector of parameters over $\mathbf{Z}_{2^{h}}$, where $\mathbf{Z}_{2^{h}}^{n}$ is a set of $n \mathrm{D}$ vectors (labels). Let

$$
\mathcal{F e}_{2}\left(\varepsilon_{2^{h}}^{c_{k}}\right):=\left[\begin{array}{rr}
1 & \varepsilon_{2^{h}}^{c_{k}} \\
1 & -\varepsilon_{2^{h}}^{c_{k}}
\end{array}\right]=\left[\begin{array}{rr}
1 & 1 \\
1 & -1
\end{array}\right]\left[\begin{array}{ll}
1 & \\
& \varepsilon_{2^{h}}^{c_{k}}
\end{array}\right], \quad k=1,2, \ldots, n,
$$

be a set of $(2 \times 2)$-matrices. Then the tensor product of these matrices

$$
\mathcal{F} \mathcal{P} \mathcal{P} g_{2^{n}}^{\left(c_{1}, c_{2}, \ldots, c_{n}\right)}:=\Delta_{0} \Pi_{2^{n}}\left(\mathcal{F}_{2}\left(\varepsilon_{2^{h}}^{c_{1}}\right) \otimes \mathcal{F}_{2}\left(\varepsilon_{2^{h}}^{c_{2}}\right) \otimes \cdots \otimes \mathcal{F}_{2}\left(\varepsilon_{2^{h}}^{c_{n}}\right)\right) \Delta_{0}
$$

gives us new multi-parametric Fourier-Prometheus transforms with fast Cooley-Tukey algorithm:

$$
\mathcal{F} \mathcal{P} \mathcal{P} \mathcal{J}_{2^{n}}^{\left(c_{1}, c_{2}, \ldots, c_{n}\right)}=\Delta_{0} \Pi_{2^{n}}\left[C T_{2^{n}}^{1}\left(\varepsilon_{2^{h}}^{c_{1}}\right) C T_{2^{n}}^{2}\left(\varepsilon_{2^{h}}^{c_{2}}\right) \cdots C T_{2^{n}}^{n}\left(\varepsilon_{2^{h}}^{c_{n}}\right)\right] \Delta_{0},
$$

where $C T_{2^{n}}^{k}\left(\varepsilon_{2^{h}}^{c_{k}}\right):=\left[I_{2} \otimes \ldots \otimes \mathcal{F}_{2}\left(\varepsilon_{2^{h}}^{c_{k}}\right) \otimes \ldots \otimes I_{2}\right]$, and $k=1,2, \ldots, n$.

\subsection{Radix-N Fourier-Prometheus transforms}

Let us consider the case of $\mathbf{Z}_{3}^{2}$. In this case $\mathcal{F C P}_{3^{2}}=\Delta_{0} \Pi_{9}\left(\mathcal{F}_{3} \otimes \mathcal{F}_{3}\right) \Delta_{0}$, where $\Delta_{0}=\operatorname{diag}\left\{\operatorname{Pr}_{(0,0)}\left(t_{1}, t_{2}\right)\right\}=\operatorname{diag}\left\{\operatorname{Pr}_{\left(t_{1}, t_{2}\right)}(0,0)\right\}$ and $\Pi_{9}$ is a special permutation matrix. From this expression we see that Prometheus functions up to constant factor are modulated Chrestenson-Clifford sequences (i.e., Clifford-valued characters of the group $\mathbf{Z}_{3}^{2}$ ):

$$
\begin{aligned}
& \operatorname{Pr}_{\left(\alpha_{1}, \alpha_{2}\right)}^{\prime}\left(t_{1}, t_{2}\right)=\operatorname{Pr}_{\left(\alpha_{1}, \alpha_{2}\right)}(0,0)\left[\operatorname{Ch}_{\left(\alpha_{1}, \alpha_{2}\right)}\left(t_{1}, t_{2}\right) \cdot \operatorname{Pr}_{(0,0)}\left(t_{1}, t_{2}\right)\right] \\
& \quad=\varepsilon_{3}^{\alpha_{1} \alpha_{2}}\left[\operatorname{Ch}_{\left(\alpha_{1}, \alpha_{2}\right)}\left(t_{1}, t_{2}\right) \varepsilon_{3}^{t_{1} t_{2}}\right]=\varepsilon_{3}^{\alpha_{1} \alpha_{2}}\left[\varepsilon_{3}^{\alpha_{1} t_{1} \oplus_{2} \alpha_{2} t_{2}} \cdot \varepsilon_{3}^{t_{1} t_{2}}\right],
\end{aligned}
$$

where

$$
\begin{aligned}
\operatorname{Pr}_{\left(\alpha_{1}, \alpha_{2}\right)}(0,0) & =\varepsilon_{3}^{\alpha_{1} \alpha_{2}} \\
\operatorname{Pr}_{(0,0)}\left(t_{1}, t_{2}\right) & =\varepsilon_{3}^{t_{1} t_{2}}
\end{aligned}
$$

and

$$
\mathrm{Ch}_{\left(\alpha_{1}, \alpha_{1}\right)}\left(t_{1}, t_{2}\right)=\varepsilon_{3}^{\alpha_{1} t_{1} \oplus_{2} \alpha_{2} t_{2}} .
$$

For this reason, this Fourier-Clifford Prometheus transform has the Cooley-Tukey fast algorithm $\mathcal{F P}_{3^{2}}=\Delta_{0} \Pi_{9}\left[C T_{9}^{1} \cdot C T_{9}^{2}\right] \Delta_{0}$, where $C T_{9}^{1}:=$ $\mathcal{F}_{3} \otimes I_{3}, C T_{9}^{2}=I_{3} \otimes \mathcal{F}_{3}$. The same result is true in the general case for Fourier-Clifford-Prometheus $\left(3^{n} \times 3^{n}\right)$-transforms

$\mathcal{F P}_{3^{n}}=\Delta_{0} \Pi_{3^{n}}\left(\mathcal{F}_{3} \otimes \mathcal{F}_{3} \otimes \cdots \otimes \mathcal{F}_{3}\right) \Delta_{0}=\Delta_{0} \Pi_{3^{n}}\left[C T_{3^{n}}^{1} C T_{3^{n}}^{2} \cdots C T_{3^{n}}^{n}\right] \Delta_{0}$, 
where $C T_{3^{n}}^{i}:=I_{3} \otimes \ldots \otimes \mathcal{F}_{3} \otimes \ldots \otimes I_{3}$ for $i=1,2, \ldots, n$ are the so-called Cooley-Tukey sparse matrices. Now we are ready to write the analogous expression for Fourier-Clifford-Prometheus $\left(N^{n} \times N^{n}\right)$-transforms

$$
\begin{gathered}
\mathcal{F P}_{N^{n}}=\Delta_{0} \Pi_{N^{n}}\left(\mathcal{F}_{N} \otimes \mathcal{F}_{N} \otimes \cdots \otimes \mathcal{F}_{N}\right) \Delta_{0} \\
=\Delta_{0} \Pi_{N^{n}}\left[C T_{N^{n}}^{1} C T_{N^{n}}^{2} \cdots C T_{N^{n}}^{n}\right] \Delta_{0},
\end{gathered}
$$

where $C T_{N^{n}}^{i}:=I_{N} \otimes \ldots \otimes \mathcal{F}_{N} \otimes \ldots \otimes I_{N}$ for $i=1,2, \ldots, n$ are the so-called Cooley-Tukey sparse matrices. The same result is true in the general case for the Fourier-Clifford-Prometheus $\left(N^{[n]} \times N^{[n]}\right)$-transform

$$
\begin{gathered}
\mathcal{F} \mathcal{P}_{N^{[n]}}=\Delta_{0} \Pi_{N^{n}}\left(\mathcal{F}_{N_{1}} \otimes \mathcal{F}_{N_{2}} \otimes \cdots \otimes \mathcal{F}_{N_{n}}\right) \Delta_{0} \\
=\Delta_{0} \Pi_{N^{n}}\left[C T_{N^{[n]}}^{1} C T_{N^{[n]}}^{2} \cdots C T_{N^{[n]}}^{n}\right] \Delta_{0},
\end{gathered}
$$

where $\Delta_{0}:=\operatorname{diag}\left(\operatorname{Pr}_{0}(t)\right)=\operatorname{diag}\left(\operatorname{Pr}_{\alpha}(0)\right)$ is a diagonal matrix and $\Pi_{N^{n}}$ a permutation matrix, and $C T_{N^{[n]}}^{i}:=I_{N_{1}} \otimes \ldots \otimes \mathcal{F}_{N_{i}} \otimes \ldots \otimes I_{N_{n}}$ for $i=1,2, \ldots, n$ are the so-called Cooley-Tukey sparse matrices.

\section{Conclusions}

We have shown how Clifford algebras can be used to formulate a new unified approach to so-called generalized Fourier-Clifford-Prometheus transforms. It is based on a new generalized FCPT-generating construction. This construction has a rich algebraic structure that supports a wide range of fast algorithms. This construction is associated not with

the triple $\left(\mathbf{Z}_{2}^{n}, \mathcal{F}_{2}, \mathbf{C}\right)$, but rather with other groups instead of $\mathbf{Z}_{2}^{n}$, other unitary transforms instead of $\mathcal{F}_{2}$, and other algebras (Clifford algebras) instead of the complex field $\mathbf{C}$.

\section{Acknowledgments}

The work was supported by the Russian Foundation for Basic Research, research project no. 03-01-00735. The paper contains some results obtained in the project no. 3258 of the Ural State Technical University.

We thank the Organizing Committee of the NATO Advanced Study Institute "Computational Noncommutative Algebra and Applications". The authors are grateful for their NATO support.

\section{References}


[1] Byrnes, J.S. (1994). Quadrature mirror filter, low crest factor arrays, functions achieving optimal uncertainty principle bounds, and complete orthonormal sequences - a unified approach. Applied and Computational Harmonic Analysis, pp. 261-264.

[2] Golay, M.J.E. ( 1949). Multislit spectrometry. J.Optical Society Am., 39:437.

[3] Golay, M.J.E. (1951). Complementary series. IRE Trans. Inform. Theory, IT-7, pp. $19-61$.

[4] Golay, M.J.E. (1977). Sieves for low autocorrelation binary sequences. IEEE Trans. Inform. Theory, IT-23, pp. 43-51.

[5] Shapiro, H.S. (1951). Extremal problems for polynomials and power series. ScM.Thesis, Massachusetts Institute of Technology.

[6] Shapiro, H.S. (1958). A power series with small partial sums. Notices of the $A M S, 6(3): 366$.

[7] Rudin, W. ( 1959). Some theorems on Fourier coefficients. Proc. Amer. Math. Soc., 10, pp. 855-859.

[8] Turan, R.J. (1974). Hadamard matrices, Baumert-Hall units, four-symbol sequences, pulse compression, and surface wave encodings. J.Combin. Theory (A). vol. 16, pp. 313-333.

[9] Eliahou, S., Kervaire, M., and Saffari, B. (1990). A new restriction on the lengths of Golay complementary sequences. J. Combin. Theory (A), vol. 55, pp. 49-59.

[10] Budisin, S.Z. (1990). New complementary pairs of sequences. Electron. Lett., Vol. 26, pp. 881-883.

[11] Sivaswamy, R. (1978). Multiphase complementary codes. IEEE Trans. Inform. Theory, vol. IT-24, pp. 546-552.

[12] Saffari, B.: History of Shapiro polynomials and Golay sequences. In preparation.

[13] Fan, P. and Darnell, M. (1996). Sequence Design for Communications Applications (Communications Systems, Technologies and Applications). Taunton, U.K.: Res. Studies,

[14] Davis, A. and Jedwab, J. (1999). Peak-to-Mean Power control in OFDM, Golay complementary Sequences, Reed-Muller codes. IEEE Trans. Inform. Theory, vol. IT-45, No. 7, pp. 2397-2417 